\title{
Ubc2, an Ortholog of the Yeast Ste50p Adaptor, Possesses a Basidiomycete-Specific Carboxy Terminal Extension Essential for Pathogenicity Independent of Pheromone Response
}

\author{
Steven J. Klosterman, Alfredo D. Martinez-Espinoza, David L. Andrews, Jeffrey R. Seay, and \\ Scott E. Gold \\ Department of Plant Pathology, University of Georgia, Athens 30602 U.S.A.
}

Submitted 19 February 2007. Accepted 26 August 2007.

\begin{abstract}
Proteins involved in the mitogen-activated protein (MAP) kinase pathway controlling mating, morphogenesis, and pathogenicity have been identified previously in the fungus Ustilago maydis. One of these, the Ubc2 adaptor protein, possesses a basidiomycete-specific structure. In addition to containing sterile $\alpha$ motif (SAM) and ras association (RA) domains typical of Ste50-like adaptor proteins found in the fungal phylum Ascomycota, Ubc2 also contains two C-terminal SH3 domains. Yeast two-hybrid assays indicated that Ubc2 interacts with the MAP kinase-kinase kinase Ubc4 via the SAM domains at each of their respective $\mathrm{N}$-termini. Site-directed mutagenesis of $u b c 2$ and complementation analyses revealed that the SAM and RA domains of Ubc2 are essential for filamentous growth. These data support a role for the ascomycete-like N-terminus of $\mathrm{Ubc} 2$ in regulating pheromone-responsive mating and morphogenesis analogous to the role of Ste50p in Saccharomyces cerevisiae. In contrast, $\mathrm{C}$-terminal deletion mutants were fully capable of filamentous growth and mating. However, surprisingly, these strains were nonpathogenic. Further, directed mutagenesis of the C-terminus revealed that both $\mathrm{SH} 3$ domains are required for pathogenicity. These results suggest that the Basidiomycota have retained the mating and morphogenetic functions of Ste50-type proteins in the N-terminal half of their Ubc2-type adaptors but, additionally, have integrated C-terminal SH3 domains that are critical for additional signal transduction mechanisms, including those that lead to pathogenesis.
\end{abstract}

Additional keywords: dimorphism, Ras.

Ustilago maydis alternates between growth as a haploid budding saprophyte and a dikaryotic filamentous pathogen. The control of this dimorphism in U. maydis has been an area of intensive study, particularly because the switch to filamentous

Corresponding author: S. E. Gold; E-mail: sgold@uga.edu

Current address of S. J. Klosterman: United States Department of Agriculture-Agricultural Research Service, 1636 Alisal St. Salinas, CA 93905, U.S.A.

Current address of A. D. Martinez-Espinoza: University of Georgia, Department of Plant Pathology, Griffin 30223, GA, U.S.A.

Current address of J. R. Seay: University of Georgia, School of Veterinary Medicine, Athens 30602, GA, U.S.A. growth is necessary for pathogenesis on maize. A number of mutations, termed $u b c$ for Ustilago bypass of cyclase, suppress the filamentous phenotype of the $U$. maydis adenylate cyclase (uac1) mutant (Gold et al. 1994; Mayorga and Gold 1998). Complementation of one of these $u b c$ suppressor mutations led to the isolation of the $u b c l$ gene encoding the regulatory subunit of cAMP-dependent protein kinase A (PKA) (Gold et al. 1997). In addition to $u b c 1$, four additional $u b c$ genes $(u b c 2, u b c 3$, $u b c 4$, and $u b c 5$ ) were cloned later by mutant complementation (Andrews et al. 2000; Mayorga and Gold 1998). The fuz7/ubc5 gene was identified earlier by homology (Banuett and Herskowitz 1994). The $u b c 3$ and $u b c 4$ genes also were identified in screens independently as $k p p 2$ and $k p p 4$, respectively (Muller et al. 1999, 2003b). The ubc3/kpp2, ubc4/kpp4, and fuz7/ubc5 genes encode a mitogen-activated protein (MAP) kinase, MAP kinase-kinase (MAPKK) kinase, and a MAPK kinase, respectively, and all are members of a pheromoneresponsive MAP kinase cascade involved in mating, morphogenesis, and pathogenic development (Andrews et al. 2000; Mayorga and Gold 1999, 2001; Muller et al. 2003b). The cAMP and MAP kinase pathways impinge on the transcription factor Prf1, which is differentially phosphorylated by PKA and the MAP kinase Ubc3/Kpp2 to activate pheromone and receptor gene expression as well as genes regulating filamentous growth (Kaffarnik et al. 2003).

Previous work revealed that $u b c 2$ encodes a novel adaptor protein involved in the pheromone-responsive MAP kinase pathway and pathogenic development on maize (Mayorga and Gold 1998, 2001). Expression of the MAPKK kinase encoded by $u b c 4$ on an autonomously replicating plasmid partially complemented a temperature-sensitive $u b c 2$ mutant, indicating a genetic and possible physical interaction between the $u b c 2$ and $u b c 4$ genes and their products, respectively (Mayorga and Gold 1998, 2001). Additionally, the $u b c 2$ mutant is morphogenetically unresponsive to mating partner-produced pheromone (Mayorga and Gold 2001). Consistent with a potential role in pheromone response, the $\mathrm{N}$-terminal half of $u b c 2$ encodes two candidate protein-protein interaction domains present in adaptor-type proteins involved in signaling and commonly found in the Ascomycota. These include a sterile $\alpha$ motif (SAM) domain and a ras association (RA) domain (Mayorga and Gold 2001). Well-studied examples of adaptor proteins containing these domains are the Schizosaccharomyces pombe and Saccharomyces cerevisiae Ste 4 and Ste50 proteins, respectively (Jansen et al. 2001; Ramachander et al. 2002). In addition to the N-terminal SAM and RA domains, the Ubc2 C-terminal 
half contains two clearly identifiable $\mathrm{SH} 3$ domains and a Ploop domain consensus.

In Ste50-like fungal adaptor proteins, the SAM domain has been studied in considerable detail. The characteristic SAM domain also is present in the adaptor Mst50 of Magnaporthe grisea, which interacts with the Mst11 MAPKK kinase required for appressorium formation and plant infection (Park et al. 2006; Zhao et al. 2005). The N-terminal SAM domain of $\mathrm{Ubc} 2$ in $U$. maydis is required for filamentous growth (Mayorga and Gold 2001), suggesting that Ubc2 plays a role similar to that of the related ascomycete adaptors in signaling morphogenesis and pathogenicity in $U$. maydis. Importantly, a SAM domain also is present in the $\mathrm{N}$-terminal region of the Ubc4/Kpp4 MAPKK kinase (Muller et al. 2003b), providing a potential mechanism of physical interaction with Ubc2, analogous to that described between Ste50p and Ste11p in S. cerevisiae. Thus, the Ubc2 protein provides a molecular handle to further probe dimorphism and, specifically, the upstream and downstream structure and function of the pheromone-responsive MAP kinase pathway in $U$. maydis.

In the current study, we analyzed the functional importance of the protein interaction domains of Ubc2 as well as Ubc2mediated protein-protein interactions. Site-directed mutagenesis and complementation analyses were carried out on $u b c 2$ to pinpoint functional domains. The findings indicate that the SAM and RA domains of Ubc 2 are essential for filamentous growth and that Ubc2 interacts with the Ubc4 MAPKK kinase via the N-terminal SAM domains present in each protein. Additionally, deletion and mutagenesis of the $\mathrm{C}$-terminal region containing the two SH3 domains in compatible strains of $U$. maydis revealed that neither of these domains is required for filamentous growth or mating. However, both are necessary for pathogenic development on maize. These data indicate not only that Ubc2 plays a dual role in signaling mating and morphogenesis but also that the basidiomycete-specific C-terminal region plays an additional key role in pathogenesis independent of pheromone response.

\section{RESULTS}

Ubc2-type adaptor proteins possess

a basidiomycete-specific architecture.

One objective of this study was to investigate the lineage of the architecture of the Ubc2-type adaptor proteins. Ubc2-related proteins of the Ascomycota share the SAM and RA domains but terminate shortly beyond the RA domain and, thus, lack the more C-terminal domains found in Ubc2 (Fig. 1). In contrast, the Ubc2-type adaptor structure is conserved among all sequenced members of the Basidiomycota, with each known or putative ortholog possessing two C-terminal SH3 domains in addition to the N-terminal SAM and RA domains (Fig. 1). The Cterminal extension beyond the RA domain in the basidiomycete adaptors represents an approximately $40 \%$ extension in length compared with the ascomycete adaptors. The available ascomycete databases were surveyed for potential orthology to the Ubc2 $\mathrm{SH} 3$ domain-containing C-terminal region. These searches did not detect any close orthologs. The zygomycete Rhizopus oryzae encodes an ortholog with the same arrangement of SAM and RA domains but, like the ascomycete proteins, lacks the C-terminal SH3 domain-containing region (Fig. 1). Of the adaptor proteins featured in Figure 1, those present in U. maydis (Mayorga and Gold 2001), Cryptococcus neoformans (S. J. Klosterman, C. B. Nichols, J. Heitman, and S. E. Gold, unpublished), M. grisea (Park et al. 2006; Zhao et al. 2005), S. cerevisiae (Jansen et al. 2001), and Schizosaccharomyces pombe (Barr et al. 1996) have established roles in the regulation of morphogenesis or pheromone-response. The $u b c 2$ homologous sequences were identified on the same supercontig in the database of the basidiomycete Puccinia graminis f. sp. tritici, which is not yet annotated for public release. In the current genome assembly, approximately $180 \mathrm{~kb}$ of sequence separates the N-terminal SAM-RA and C-terminal SH3 domain coding regions on this supercontig. A stretch of misreads immediately follows the region encoding the N-terminal SAM-RA domains; thus, it is plausible that the region of this supercontig is misassembled.

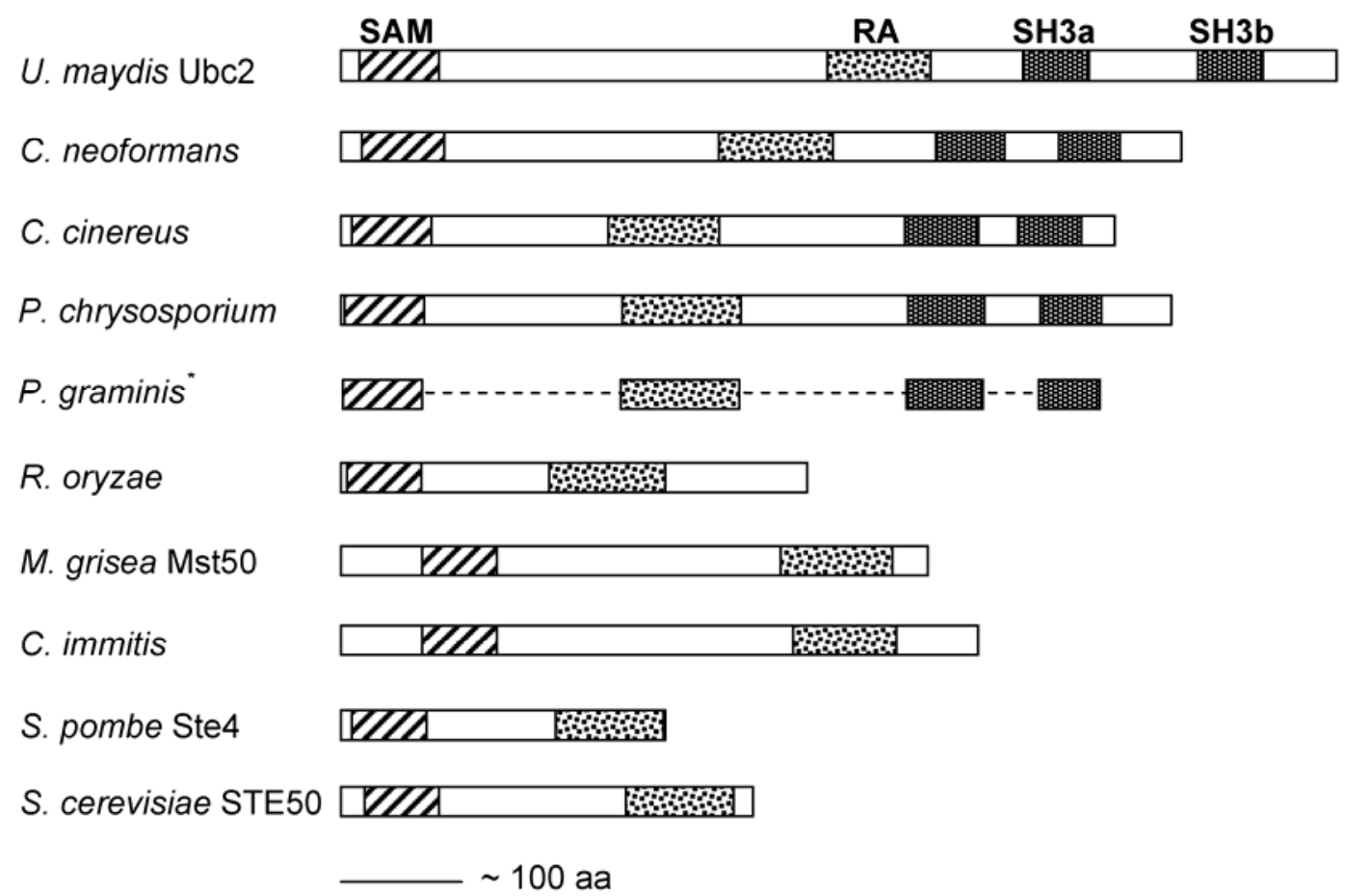

Fig. 1. The N-terminus of Ubc2 is conserved throughout the fungi, whereas the C-terminus is Basidiomycota specific. Representative Ubc2-related fungal adaptor proteins. The positions of the sterile- $\alpha$-motif (SAM), ras association (RA), and two src homology 3 (SH3) domains are shown. The scale is approximate and intended to show the overall structures of these proteins. 
To explore the phylogenetic relationship among the Ubc2type adaptor proteins, a distance tree was generated using regions of the SAM and RA domains present in 20 of these orthologous proteins (Fig. 2). The distance tree demonstrates conservation of these sequences in three Phyla tested. The representatives of the Basidiomycota, including $U$. maydis and three divergent Hymenomycetes, form a distinct clade at the top of this tree (Fig. 2). The filamentous Ascomycota, including M. grisea and Aspergillus nidulans, cluster in the middle of this tree as a sister clade to the members of the Basidiomycota (Fig. 2). The yeast-like and dimorphic Hemiascomyctes and Archeascomycetes scatter in the lower portion of this tree (Fig. 2). Interestingly, although lacking the signature basidiomycete C-terminal extension, the $R$. oryzae SAM-RA region, representative of the sole sequenced member of the Zygomycota, clusters with those of the Basidiomycota (Fig. 2).

The N-terminal SAM and RA domains are required whereas the C-terminal basidiomycete-specific extension of Ubc2 is dispensable for filamentous growth.

We initially investigated whether the putative protein-protein interacting domains of Ubc2 are necessary for morphogenesis. For this investigation, the $u a c 1 u b c 2$ double mutant strain 5/17 was employed in filamentation complementation analysis (Mayorga and Gold 2001) (Table 1). Strain 5/17 grows as a yeast because deletion of the $u b c 2$ gene suppresses the filamentous phenotype of the uacl mutant strain. Reintroduction

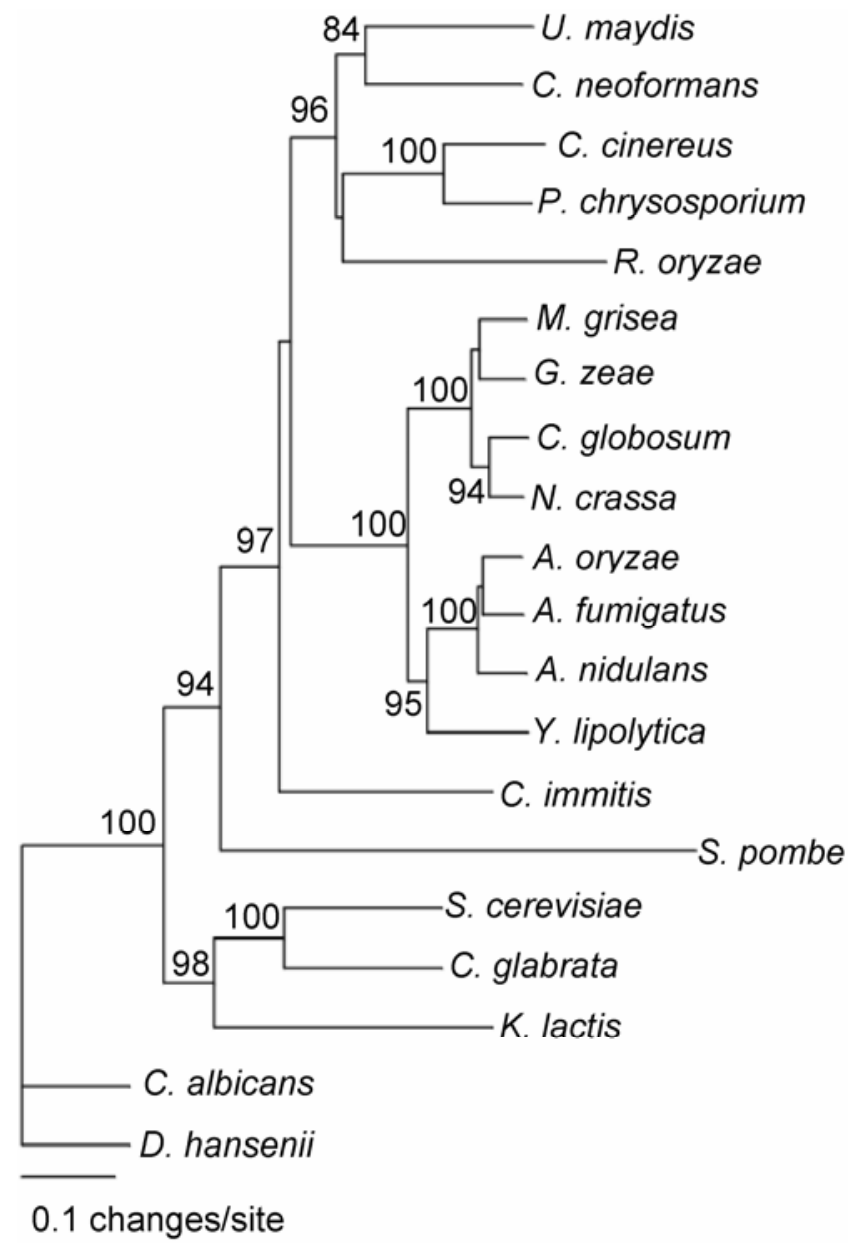

Fig. 2. Most parsimonious distance tree of the sterile- $\alpha$-motif (SAM), ras association (RA) domain regions derived from 20 related fungal proteins. Bootstrap values $>70 \%$ from 100 bootstrap replicates are indicated at the appropriate nodes. The scale bar indicates the average number of substitutions per site. of wild-type $u b c 2$ into strain $5 / 17$ produces a filamentous strain showing the uacl phenotype. Various mutations were introduced in $u b c 2$, which was residing in the autonomously replicating plasmid pSAC7, and the individually mutated plasmids (Fig. 3A) were transformed into strain 5/17. Transformation of strain 5/17 with the empty vector pHyg101 resulted in yeast colonies typical of the uncomplemented double mutant (Fig. 3B, pHyg101). Transformation with unmodified pSAC7 containing the wild-type $u b c 2$ gene resulted in complementation to filamentous growth characteristic of the single uacl mutant (Fig. 3B, pSAC7). Transformation of pSAC7 derivative plasmids harboring $u b c 2$ mutations that rendered them defective in complementation resulted in yeast colonies characteristic of the uncomplemented double mutant (Fig. 3B).

Mutagenesis and complementation analyses (Fig. 3) indicated that specific amino acids within the SAM and RA domains of Ubc2 are essential for the function of Ubc2 in filamentation. Mutagenesis of three conserved amino acids in the N-terminal SAM domain (W17A, V23A, and W25A) in a single plasmid prevented complementation (Fig. 3A, site-directed mutant 1, and $\mathrm{B}$, colony 1$)$. Three amino acid changes introduced into the RA domain (D443A, D444A, and Y448A) in a single plasmid also abolished complementation (Fig. 3A, site-directed mutant 3 , and $\mathrm{B}$, colony 3 ). Microscopically, strains harboring the plasmid mutants 1 and 3 (Fig. 3B) appeared morphologically typical of the original $u a c 1 u b c 2$ double mutant.

To investigate the role of the basidiomycete-specific domains housed in the C-terminal half of Ubc2, we constructed missense and nonsense mutations in this region. Mutations introduced into either of the regions encoding the $\mathrm{SH} 3$ domains had no effect on $u b c 2$ function in filamentation (Fig. 3A, site-directed mutants 5 to 9 , and $\mathrm{B}$, colonies 5 to 9). Additionally, deletion of the P-loop consensus had no effect on filament complemen-

Table 1. Strains of Ustilago maydis used in this study

\begin{tabular}{|c|c|c|}
\hline Strain & Relevant genotype $^{a}$ & Source \\
\hline $1 / 2$ & alb1 & Gold et al. 1997 \\
\hline $2 / 9$ & $\begin{array}{l}a 2 b 2(\mathrm{BX} 7 \mathrm{~A} 22, \text { near isogenic to } \\
1 / 2)\end{array}$ & Gold et al. 1997 \\
\hline $5 / 9$ & 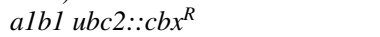 & Mayorga and Gold 2001 \\
\hline $5 / 10$ & $a 2 b 2 u b c 2: \because c b x^{R}$ & Mayorga and Gold 2001 \\
\hline $15 / 5$ & $\begin{array}{l}\text { alb1 ubc2Cterminus::cbx }{ }^{R}+ \\
\text { pSAC7 } \triangle \mathrm{P}-\text { loop }^{*}\end{array}$ & This study \\
\hline $15 / 6$ & $\begin{array}{l}\text { a2b2 ubc2Cterminus::cbx }{ }^{R}+ \\
\text { pSAC7 } \triangle \mathrm{P}-\text { loop* }\end{array}$ & This study \\
\hline $15 / 7$ & $\begin{array}{l}\text { alb1 ubc2Cterminus::cbx }{ }^{R}+ \\
\text { pSAC7 } \triangle \mathrm{SH} 3 \mathrm{a}^{*}\end{array}$ & This study \\
\hline $15 / 8$ & $\begin{array}{l}\text { a2b2 ubc2Cterminus::cbx }{ }^{R}+ \\
\text { pSAC7 } \triangle \text { SH3a* }\end{array}$ & This study \\
\hline $5 / 17$ & 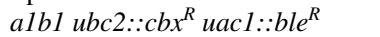 & Mayorga and Gold 2001 \\
\hline $15 / 9$ & alb1 ubc2Cterminus::cbx $x^{R}$ & This study \\
\hline $15 / 10$ & $a 2 b 2$ ubc2Cterminus:: $c b x^{R}$ & This study \\
\hline $15 / 11$ & $\begin{array}{l}\text { alb1 ubc2Cterminus::cbx }{ }^{R}+ \\
\text { pSAC7 } \triangle \mathrm{SH}^{2} \mathrm{~b}^{*}\end{array}$ & This study \\
\hline $15 / 12$ & $\begin{array}{l}\text { a2b2 ubc2Cterminus:: } c b x^{R}+ \\
\text { pSAC7 } \triangle \mathrm{SH}^{*} \mathrm{~b}^{*}\end{array}$ & This study \\
\hline $15 / 13$ & $\begin{array}{l}\text { alb1 ubc2Cterminus::cbx } x^{R}+ \\
\text { pSAC7* }\end{array}$ & This study \\
\hline $15 / 14$ & $\begin{array}{l}\text { a2b2 ubc2Cterminus:: } c b x^{R}+ \\
\text { pSAC7* }\end{array}$ & This study \\
\hline $15 / 23$ & $\begin{array}{l}\text { a2b2 ubc2Cterminus:: } c b x^{R}+ \\
\text { pSAC7GP(SH3b)* }\end{array}$ & This study \\
\hline $15 / 24$ & $\begin{array}{l}\text { a2b2 ubc2Cterminus:: } c b x^{R}+ \\
\text { pSAC7SY }(\mathrm{SH} 3 \mathrm{~b})^{*}\end{array}$ & This study \\
\hline $15 / 25$ & $\begin{array}{l}a 2 b 2 \text { ubc2Cterminus:: } c b x^{R}+ \\
\text { pSAC7YY }(\mathrm{SH} 3 \mathrm{a})^{*}\end{array}$ & This study \\
\hline $15 / 26$ & $\begin{array}{l}\text { a2b2 ubc2Cterminus::cbx } x^{R}+ \\
\text { pHyg } 101^{*}\end{array}$ & This study \\
\hline
\end{tabular}

${ }^{a}$ An asterisk (*) indicates integrated plasmid. 
tation (Fig. 3B, colony 10). To investigate further whether the SH3a, SH3b, P-loop, or other unidentified domains play roles in facilitating filamentous growth, stop codons were introduced into $u b c 2$ at positions immediately following the region encoding the RA domain (K492stop, S493stop). Introduction of this plasmid into strain $5 / 17$ still resulted in typical wild-type $u b c 2$ complementation to the filamentous colony phenotype (Fig. 3B, colony 11). Finally, deletion of the entire SH3 domaincontaining C-terminus in a separate construct and its introduction into strain $5 / 17$ still yielded a plasmid that fully complemented to filamentous growth (not shown). This construct was different than the construct produced for colony 11 (Fig. 3B) in that the complete $\mathrm{C}$-terminal-containing region was replaced with the carboxin resistance gene. Thus, all mutant $u b c 2$ alleles modified within the $\mathrm{C}$-terminus were unaltered in their ability to complement the filamentation growth defect in the $u a c 1 u b c 2$ double mutant. These results indicated that the $\mathrm{N}$-terminal half of Ubc2, containing the SAM and RA domains, was sufficient for complementation of filamentous growth and, importantly, that the basidiomycete-specific C-terminus was completely dispensable for this function.

\section{The C-terminal SH3 domain-containing region} of $\mathrm{Ubc} 2$ is not necessary for mating.

To clarify the role of the C-terminal SH3 domain-containing region of Ubc2 in mating, $u b c 2$ C-terminal deletion gene

A

\begin{tabular}{|c|c|c|c|c|c|}
\hline SAM & & RA & SH3a & $\mathrm{SH} 3 \mathrm{~b}$ & ploop \\
\hline$B D D D$ & Ubc2 & & & & \\
\hline
\end{tabular}

Truncated Ubc2

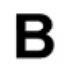

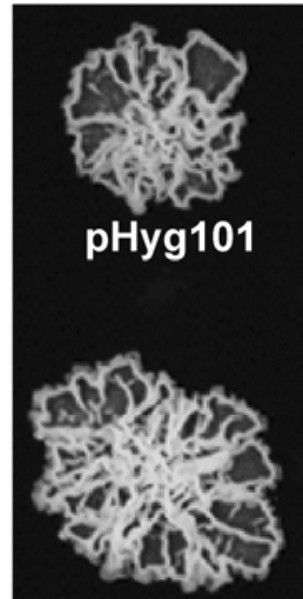

3

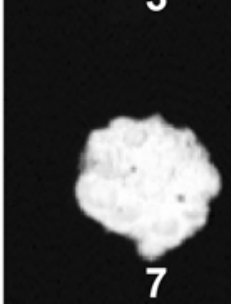

7

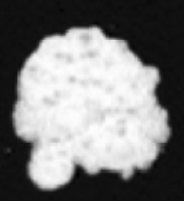

4
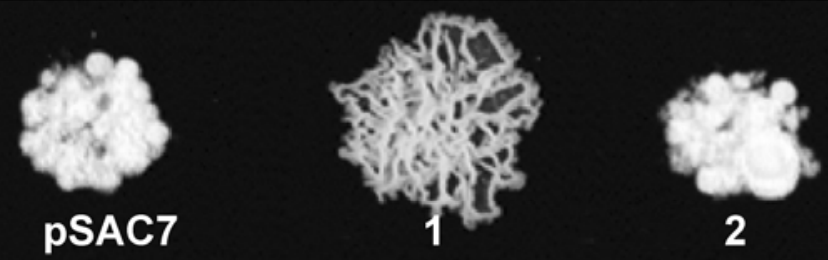

2

11
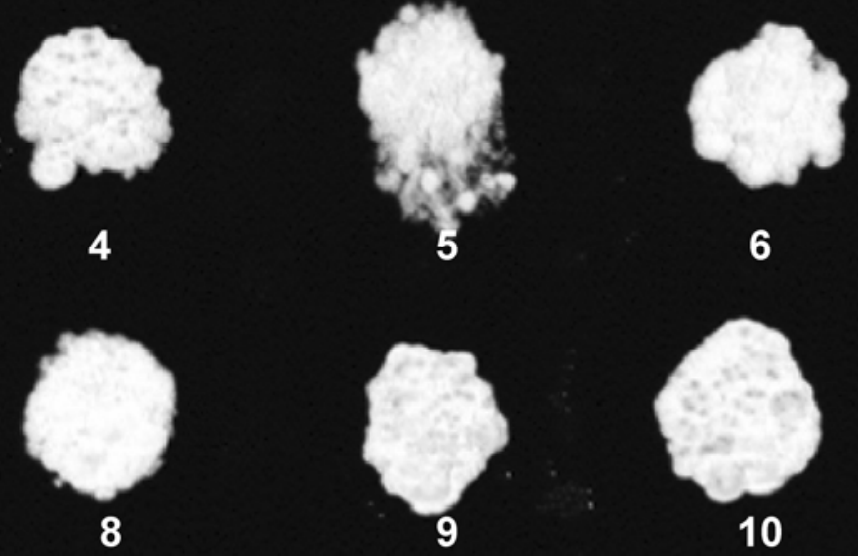

6

5
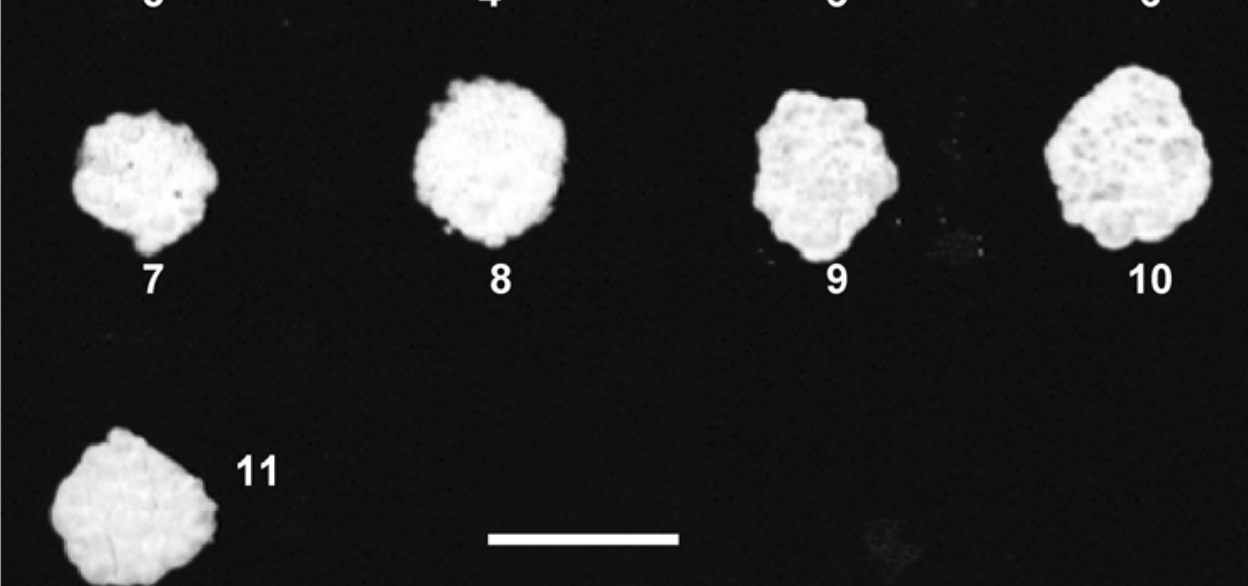

Fig. 3. Site-directed mutagenesis of $u b c 2$ and complementation analyses. A and $\mathbf{B}$, Numbers 1 to 11 correspond to locations and respective phenotypes of specific mutants in the Ubc2 protein. A, Mutations 1 to 10 introduced missence mutations while mutation 11 generated two adjacent nonsense mutations and, thus, truncation of the encoded protein immediately following the ras association (RA) domain. SAM = sterile- $\alpha$-motif and SH3 = src homology 3 . B, Strain 5/17 was transformed with the negative and positive complementation control plasmids pHyg101 or pSAC7, respectively, or with the site-directed mutagenized constructs. The solid white filamentous colony morphology is indicative of complementation equivalent to wild-type Ubc2 while the ruffled gray appearance of colonies 1 and 3 is indicative of the uncomplemented budding phenotype of the Ustilago maydis uac1 ubc2 double mutant strain $5 / 17$. The morphological phenotypes of the transformants were analyzed by plating on potato dextrose agar medium containing hygromycin B at $150 \mu \mathrm{g} / \mathrm{ml}$ for plasmid maintenance and $1 \%$ charcoal for visual contrast. Scale bar $=1 \mathrm{~cm}$. 
replacement mutant strains were produced in two mating-compatible wild-type strains (15/9 and 15/10). Deletions were confirmed by DNA blot analysis (not shown). Mating of these compatible $u b c 2 \mathrm{C}$-terminal mutant strains led to the production of white filamentous colonies typical of strong mating reactions that were comparable with those of wild-type strains 1/2 and 2/9 (Fig. 4). This result showed that the truncated Ubc 2 protein containing the N-terminal 491 amino acids is expressed because it complemented the mating defect of the $u b c 2$ deletion strain. This observation that the C-terminal truncation of Ubc2 did not play a major role in mating suggested that this region may function in signaling in the Phylum Basidiomycota distinct from the Ste50p-type adaptor proteins in the Ascomycota.

\section{The C-terminal SH3 domain-containing region of $\mathrm{Ubc} 2$ is required for pathogenicity.}

Because the Ubc2 C-terminal region had no apparent role in mating or filamentation, we investigated its possible role in pathogenesis. To investigate the role of the $\mathrm{C}$-terminal region of Ubc2 in pathogenicity, maize seedlings were inoculated with $u b c 2$ C-terminal mutant strains $15 / 9$ and 15/10 and compared with those plants inoculated with wild-type strains $1 / 2$ and 2/9 (Table 2). Pathogenicity was compromised in complete $u b c 2$ deletion strains 5/9 and 5/10 (Mayorga and Gold 2001), included as controls (Table 2). Pathogenicity assays indicated that the C-terminal region of $\mathrm{Ubc} 2$, including the two $\mathrm{SH} 3$ domains, is required for pathogenic development because no galls or other symptoms were observed in plants inoculated with compatible C-terminal deletion mutant strains 15/9 and $15 / 10$ (Table 2). When either strain $15 / 9$ or $15 / 10$ was co-inoculated with the compatible wild-type partner, disease progression was comparable with that of the wild-type cross (not shown), indicating that a single wild-type allele is sufficient for restoration of pathogenicity. This corroborates previous findings, in which $u b c 2$ null mutants crossed with compatible wild-type strains resulted in pathogenesis equivalent to wild-type crosses (Mayorga and Gold 2001). To verify the role of the SH3 domain-containing C-terminal region of Ubc2 in pathogenesis, assays for complementation of pathogenicity function were conducted with the wild-type $u b c 2$ allele. For this purpose,

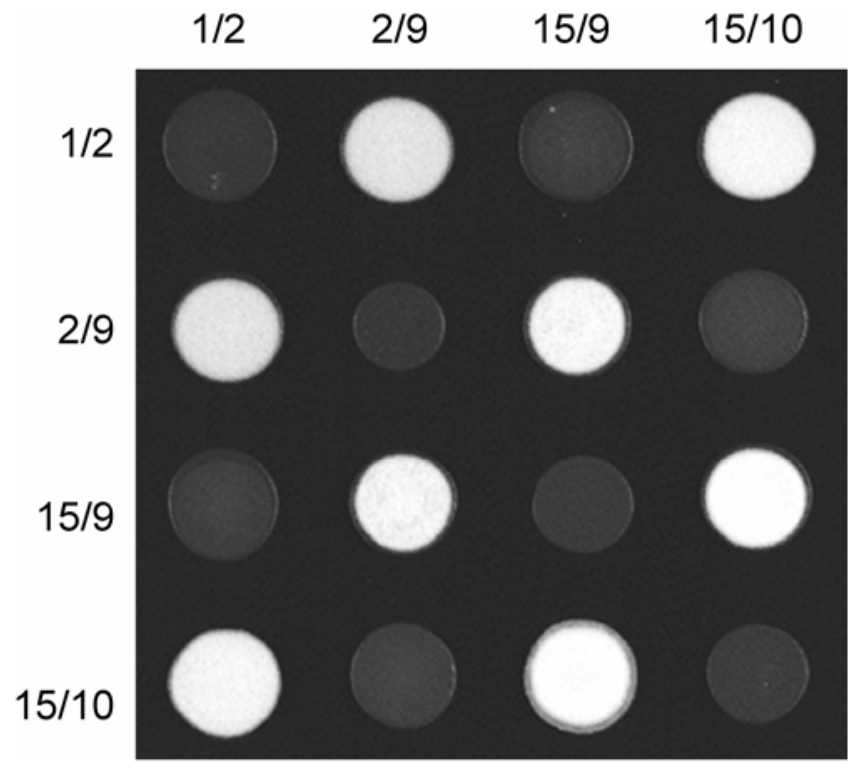

Fig. 4. Ubc2 src homology 3 (SH3) domain-containing carboxy terminus is dispensable for mating. The analysis includes compatible wild-type strains $1 / 2$ and $2 / 9$ and $u b c 2$ carboxy terminal deletion mutant strains $15 / 9$ and $15 / 10$. White filamentous colonies are indicative of mating at $48 \mathrm{~h}$. linearized plasmid $\mathrm{pSAC7}$, lacking the autonomous replication sequence (ARS) and carrying the wild-type $u b c 2$ allele, was integrated into the $u b c 2$ C-terminal deletion mutants $15 / 9$ and $15 / 10$. Presence of wild-type $u b c 2$ in these strains was verified by polymerase chain reaction (PCR). Complementation with the wild-type $u b c 2$ allele restored pathogenicity to the $u b c 2 \mathrm{C}$ terminal deletion mutant strains to levels comparable with those observed in wild-type inoculations (Table 2).

\section{Both C-terminal SH3 domains of Ubc2 are required for pathogenesis.}

To more fully explore the function of the C-terminus of Ubc2, we determined whether SH3a, SH3b, or the P-loop consensus encoding regions of $u b c 2$ were necessary for pathogenesis. To accomplish this aim, several domain-specific $u b c 2$ deletion mutants were prepared in pSAC7 and introduced into Cterminal mutant strains $15 / 9$ and 15/10 (Table 1).

The resulting transformants then were assayed for complementation of pathogenicity function. Deletion of a group of nine codons encoding a block of more highly conserved amino acids in SH3a (Fig. 1), followed by transformation with this construct, resulted in strains $15 / 7$ and 15/8 (Table 1). Crosses of these mutants were nonpathogenic (Table 2). Similarly, deletion of a group of 10 codons encoding a block of more highly conserved amino acids in $\mathrm{SH} 3 \mathrm{~b}$ of $\mathrm{Ubc} 2$ resulted in strains $15 / 11$ and $15 / 12$ that were also nonpathogenic (Table $2)$. However, deletion of the entire P-loop region, and complementation with this construct, resulted in strains (15/5 and $15 / 6$ ) that, when inoculated together, exhibited pathogenicity comparable with wild-type crosses (Table 2).

To examine whether specific conserved amino acids in $\mathrm{SH}$ a and $\mathrm{SH} 3 \mathrm{~b}$ of $\mathrm{Ubc} 2$ were important in pathogenicity, the sitedirected mutant pSAC7 derivative plasmids used in the filamentation complementation analyses of $u b c 2$ were used in pathogenicity complementation experiments (Table 3). The plasmids were linearized and transformed into strains 15/9 and $15 / 10$ for pathogenicity complementation analyses. In each of the inoculations, these strains were mixed with the compatible mating strain of the $u b c 2 \mathrm{C}$-terminal deletion mutant. Thus, in each cross, only one potentially complementing allele (for pathogenicity) was present. These analyses revealed that at least two conserved amino acids in each of the C-terminal SH3 domains of Ubc2 are required for pathogenicity and provide further evidence that both $\mathrm{SH} 3$ domains are necessary for pathogenicity. The site-directed mutant pair Y572A, Y574A in

Table 2. Pathogenicity of $u b c 2$ C-terminal deletion mutants ${ }^{\mathrm{a}}$

\begin{tabular}{|c|c|c|c|c|c|}
\hline $\begin{array}{l}\text { Inoculum } \\
\text { (strains) }\end{array}$ & $u b c 2$ Status ${ }^{b}$ & $\begin{array}{l}\text { No. of } \\
\text { plants }\end{array}$ & $\begin{array}{l}\text { Chlorosis, } \\
\text { anthocyanin }^{\text {d }}\end{array}$ & $\begin{array}{c}\text { Plants } \\
\text { with galls }\end{array}$ & Death \\
\hline $1 / 2 \times 2 / 9$ & $\begin{array}{l}\text { Wild-type } \\
u b c 2\end{array}$ & 60 & 5 & 50 & 4 \\
\hline $5 / 9 \times 5 / 10$ & $\Delta u b c 2$ & 60 & 0 & 0 & 0 \\
\hline $15 / 9 \times 15 / 10$ & $\Delta \mathrm{C}$-term & 60 & 0 & 0 & 0 \\
\hline $15 / 14 \times 15 / 13$ & $\begin{array}{l}\Delta \text { C-term- } \\
\text { comp }^{\mathrm{e}}\end{array}$ & 60 & 6 & 49 & 4 \\
\hline $15 / 7 \times 15 / 8$ & $\triangle \mathrm{SH} 3 \mathrm{a}^{\mathrm{f}}$ & 60 & 0 & 0 & 0 \\
\hline $15 / 11 \times 15 / 12$ & $\Delta \mathrm{SH} 3 \mathrm{~b}$ & 60 & 0 & 0 & 0 \\
\hline $15 / 5 \times 15 / 6$ & $\Delta \mathrm{P}$-loop & 60 & 10 & 46 & 2 \\
\hline
\end{tabular}

${ }^{a}$ Symptom development 10 days after inoculation.

${ }^{\mathrm{b}}$ Domain modified or deleted; C-term = C-terminal deletion mutant of $u b c 2$.

${ }^{\mathrm{c}}$ Total number of plants $=$ combined data from three independent experiments of 20 plants each.

d Plants with chlorosis, anthocyanin, or both.

e The $u b c 2$ C-terminal deletion strains complemented with linearized plasmid pSAC7 containing wild-type $u b c 2$.

${ }^{\mathrm{f}}$ In this case, $\Delta$ means deletion of selected codons in $\mathrm{SH} 3 \mathrm{a}$, and the same applies for $\mathrm{SH} 3 \mathrm{~b}$. 
Table 3. Pathogenicity of $u b c 2$ C-terminal site-directed mutants ${ }^{\mathrm{a}}$

\begin{tabular}{|c|c|c|c|c|c|}
\hline $\begin{array}{l}\text { Inoculum } \\
\text { (strains) }\end{array}$ & $u b c 2$ Status $^{b}$ & $\begin{array}{l}\text { No. of } \\
\text { plants }\end{array}$ & $\begin{array}{l}\text { Chlorosis, } \\
\text { anthocyanin }^{d}\end{array}$ & $\begin{array}{c}\text { Plants } \\
\text { with galls }\end{array}$ & Death \\
\hline $1 / 2 \times 2 / 9$ & $\begin{array}{l}\text { Wild-type } \\
u b c 2\end{array}$ & 60 & 5 & 50 & 2 \\
\hline $5 / 9 \times 5 / 10$ & $\Delta u b c 2$ & $40^{\mathrm{e}}$ & 0 & 0 & 0 \\
\hline $15 / 9 \times 15 / 10$ & $\Delta \mathrm{C}$-term & 60 & 0 & 0 & 0 \\
\hline $15 / 13 \times 15 / 14$ & $\begin{array}{l}\Delta \text { C-term- } \\
\text { comp }^{\text {f }}\end{array}$ & 60 & 14 & 28 & 1 \\
\hline $15 / 9 \times 15 / 26$ & $\Delta \mathrm{C}$-term $\mathrm{g}$ & 60 & 0 & 0 & 0 \\
\hline $15 / 9 \times 15 / 25$ & YYSH3a & 60 & 0 & 0 & 0 \\
\hline $15 / 9 \times 15 / 24$ & SYSH3b & 60 & 18 & 33 & 0 \\
\hline $15 / 9 \times 15 / 23$ & GPSH3b & 60 & 0 & 0 & 0 \\
\hline
\end{tabular}

a Symptom development 10 days after inoculation.

${ }^{\mathrm{b}}$ Domains modified; C-term $=\mathrm{C}$-terminal deletion mutant of $u b c 2$.

$\mathrm{c}$ Total number of plants $=$ combined data from three independent experiments of 20 plants each.

d Plants with chlorosis, anthocyanin, or both.

e Combined data from two independent experiments of 20 plants each.

${ }^{\mathrm{f}} \mathrm{C}$-terminal deletion mutant of $u b c 2$ complemented with wild-type $u b c 2$.

g Complementation negative control; $u b c 2$ C-terminal deletion mutant strains transformed with the integrated empty vector pHyg 101.

${ }^{\mathrm{h}}$ YYSH3a designates two codons specifying tyrosine that were mutagenized in the region encoding $\mathrm{SH} 3$ domain a $(\mathrm{SH} 3 \mathrm{a})$ of $u b c 2$. Similar designations are included for the mutagenized $\mathrm{SH} 3 \mathrm{~b}$ domain. domain SH3a and present in strain 15/25 did not complement for pathogenicity function. Similarly, the site-directed mutant pair of changes G755A, P758A in domain SH3b in strain $15 / 23$ did not complement for pathogenicity. In contrast, the SH3b mutant pair S759A, Y761A, representing two less conserved amino acids, complemented to levels approaching wild type for pathogenicity (strain 15/24×15/9) (Table 3 ).

\section{Ubc2 physically interacts with the Ubc4 MAPKK kinase.}

To determine whether the Ubc2 protein forms homodimers or interacts with candidate G-proteins or members of the MAP kinase cascade controlling mating, morphogenesis, and pathogenicity, directed yeast two-hybrid analyses were performed using full-length Ubc2 and Ubc4 as well as variants of Ubc2 and Ubc4 as bait (Fig. 5A). Ubc2, Ubc3, Ubc4, Ubc5, Ras1, and Ras2 protein fusions were used as prey in these experiments (Fig. 5B). Immunoblotting and in vitro translated $\mathrm{S}^{35}$-labeled protein confirmed the presence of the fusion proteins at the expected molecular weights (not shown). Positive interactions were determined by the growth of yeast colonies that were blue on synthetic dropout medium supplemented with X$\alpha$-gal. Robust growth and blue coloration are characteristic of reporter gene expression and an indirect assay for the interactions between bait and prey proteins using this system. Co-trans-

A

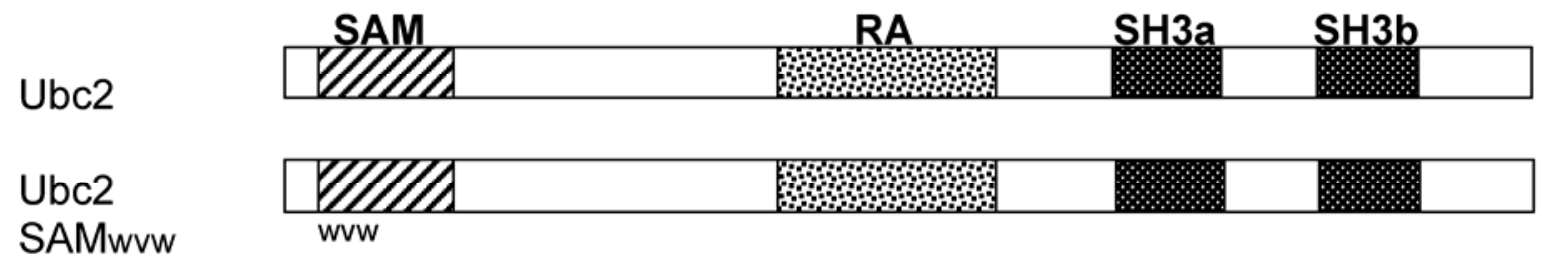

Ubc2SAMRA WOZM

Ubc2SAM

Ubc2RASH3

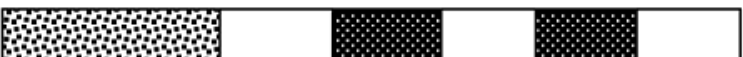

B

\begin{tabular}{llccccccc}
\multicolumn{1}{c}{ Bait } & Ubc2 & Ubc4 & Ubc4SAM & Ubc3 & Ubc5 & Ras1 & Ras2 \\
\cline { 2 - 5 } & - & + & + & - & - & - & - \\
& Ubc4 & + & - & - & - & - & - & - \\
Ubc2SAMRA & - & + & + & $-*$ & $-*$ & $-*$ & $-*$ \\
& Ubc2SAM & - & + & + & $-*$ & $-*$ & $-*$ & $-*$ \\
Ubc2RASH3 & - & - & - & $-*$ & $-*$ & $-*$ & $-*$ \\
Ubc2SAMwvw & - & - & - & - & - & - & - \\
\hline
\end{tabular}

Fig. 5. Pairwise yeast two-hybrid assays using Ubc2 and Ubc4 as bait. A, Ubc2 and the mutant or truncated versions of Ubc2 as bait protein used in this study. The "wvw" below the region of $u b c 2$ encoding the sterile- $\alpha$-motif (SAM) domain indicates mutations at three codons: W17A, V23A, and W25A. RA = ras association and SH3 = src homology 3. B, Results of the pairwise yeast two-hybrid assays. Yeast reporter strain AH109 was co-transformed with the pGBKT7 bait and pGADT7 prey plasmids encoding the bait and prey proteins as fusions with the GAL4 DNA binding domain or the GAL4 activation domain, respectively. Positives (+) are based on growth of yeast colonies and blue coloration on synthetic dropout medium (-Ade/-His) overlayed with X- $\alpha$-gal. Each of the experiments designated as + or - were repeated at least three times and Western blots (not shown) were performed to confirm expression of each protein at the appropriate molecular weight. Those experiments marked by -* were negative for colony growth and were repeated twice. The pGBKT7p53 bait plasmid gave consistently positive results when coupled with the positive control plasmid pGADT7-T-antigen in cotransformation experiments (not shown). The negative control plasmid pGADT7-Lam gave consistently negative results when cotransformed with the pGBKT7-p53 bait plasmid (not shown). 
formation of plasmids encoding the full-length, wild-type Ubc2 and full-length Ubc4 fusion proteins resulted in yeast colony growth and blue coloration (Fig. 5B). Additionally, when the plasmid encoding the SAM domain fragment of Ubc2 (amino acids 1 to 77) was co-transformed with plasmids encoding the full-length Ubc4 or a Ubc4 protein fragment bearing only the SAM domain (Ubc4SAM, amino acids 1 to 120), colony growth and blue coloration were observed. The bait plasmid encoding the "SAMRA" (Fig. 5) fusion (amino acids 1 to 491) also resulted in blue colony growth when co-transformed with plasmids encoding either the full-length Ubc4 or the SAM domain-containing fragment of Ubc4. In contrast, yeast colonies were not observed when a plasmid encoding the Ubc2 RA domain and two C-terminal SH3 domains ("RASH3", amino acids 410 to 829) (Fig. 5), and thus lacking the N-terminal SAM domain region, was co-transformed into yeast together with full-length Ubc4. Again, no growth was observed when full-length Ubc2, carrying mutations in three conserved SAM domain amino acids, was used as bait with the full-length Ubc4 fusion as prey. When plasmids encoding the Ubc2 or Ubc4 protein fusions were co-transformed with the Ubc3, Ubc5, Ras1, or Ras2 fusions, yeast growth was not observed (Fig. 5B). Coimmunoprecipitation was used to verify the interaction between antibody tagged Ubc 2 and Ubc 4 proteins in vitro. The tagged Ubc2 protein co-precipitated with Ubc4 when either the anti-HA (Ubc4 tag) or anti-Myc (Ubc2 tag) antibodies were used in the precipitation analyses (not shown). However, the tagged RASH3 fragment of the Ubc2 protein lacking the $\mathrm{N}$-terminal region, including the SAM domain, did not co-precipitate with the tagged Ubc4 protein (not shown). These results indicated that, analogous to other fungal systems studied, the SAM domain of the adaptor protein Ubc2 directly interacts with the SAM domain of its cognate MAPKK kinase, Ubc4. No other Ubc2 interactors were detected in these directed twohybrid assays.

\section{DISCUSSION}

The $U$. maydis $u b c 2$ gene was identified as a genetic suppressor required for filamentous growth (Mayorga and Gold 1998). The full structure of Ubc2, bearing two C-terminal SH3 domains in addition to the SAM and RA N-terminal domains, led to the hypothesis that Ubc2 may act as a novel adaptor protein facilitating protein-protein interactions both in the conserved roles of mating and filamentation via its $\mathrm{N}$-terminal domains and in new, additional functions via its C-terminal SH3 domains (Mayorga and Gold 2001). In this study, filamentation complementation assays revealed the N-terminal SAM and RA domains are required for filamentous growth whereas the C-terminal SH3 domains and P-loop consensus are not required for filamentous growth. Yeast two-hybrid analyses indicated interaction between the SAM domains of Ubc2 and Ubc4. Complementation of pathogenicity function with mutant versions of $u b c 2$ revealed that both $\mathrm{SH} 3$ domains are necessary for pathogenicity.

Structurally, the Ubc 2 protein possesses a clear relationship to adaptor proteins found in the Ascomycota, such as Ste50p of Saccharomyces cerevisiae (Mayorga and Gold 2001). Exploration of the available basidiomycete genome databases indicated that the overall architecture of Ubc2 is conserved among orthologs in the Basidiomycota. The genome of the basidiomycete $P$. graminis f. sp. tritici is not yet annotated for public release. Nevertheless, a search of the $P$. graminis f. sp. tritici database revealed sequences sharing homology with $u b c 2$ on the same supercontig. Approximately $180 \mathrm{~kb}$ of sequence separates the N-terminal SAM-RA and C-terminal SH3 domain coding regions on this supercontig. This discrepancy may reflect misassembly in this region of the supercontig and further genome annotation likely will clarify this matter. The zygomycete $R$. oryzae possesses an ortholog with the same arrangement of SAM and RA domains but, like the ascomycete orthologs, it lacks the C-terminal SH3 domain-containing region. The Zygomycota is considered evolutionarily basal to both the Ascomycota and Basidiomycota (Guarro et al. 1999); therefore, this suggests that the region encoding the C-terminus of the Ubc2 protein was an addition coincident with or following the divergence of Basidiomycota and Ascomycota from a common ancestor. Because the C-terminal extension is present across all of the divergent basidiomycetes examined, the appearance of the $\mathrm{SH} 3$ domain-containing structure likely occurred at least 400 million years ago. A distance tree of the SAM and RA domain regions indicates a closer relationship of the sole representative $\mathrm{Ubc} 2$ ortholog of the Zygomycota to those of the Basidiomycota than to those of the Ascomycota. Thus, based on this necessarily limited data set, the SAM and RA domains of the basidiomycete adaptors apparently retained more in common with those of the ancestral proteins while orthologs in the Ascomycota appear more diverged. Database searches did not reveal a protein that bears close orthology with the Ubc2 $\mathrm{C}$-terminal region in organisms other than the Basidiomycota. Therefore, the derivation of the C-terminal SH3 domains in the basidiomycete-specific Ubc2-like adaptors remains unclear.

Like the well-studied $S$. cerevisiae Ste 50p, Ubc2 possesses a $\mathrm{N}$-terminal SAM domain, a conserved protein-binding domain of approximately 70 amino acids present as a single copy in proteins (Ponting 1995). In S. cerevisiae, Ste50p and the Stellp MAPKK kinase interact via their N-terminal SAM domains, thereby facilitating pseudohyphal growth and pheromoneresponsive mating (Jansen et al. 2001). Similarly, the Schizosaccharomyces pombe Ste4 adaptor and the Byr2 MAPKK kinase interact via their SAM domains, also facilitating morphogenic changes (Barr et al. 1996; Ramachander et al. 2002). Park and associates (2006) showed that the related adaptor MST50 from the rice blast pathogen $M$. grisea has extensive interactions with signaling components in the PMK1 MAP kinase cascade. Like the other Ste50p-like proteins mentioned above, MST50 interacts with MST11, the MAPKK kinase, via their respective SAM domains (Park et al. 2006).

In previous work, the precise deletion of the SAM domain of Ubc2 showed that it was essential for filamentous growth (Mayorga and Gold 2001). Moreover, a temperature-sensitive SAM domain mutant (K78E) was identified that allowed this strain to grow filamentously at the permissive temperature of $18^{\circ} \mathrm{C}$ and in the budding form at the nonpermissive temperature of $30^{\circ} \mathrm{C}$ (Mayorga and Gold 2001). In the present study, site-directed mutagenesis experiments revealed that a particular set of mutations in conserved residues of the Ubc2 SAM domain (W17A, V23A, and W25A) abrogate filamentation complementation of the budding uacl $u b c 2$ double mutant. These three amino acids, although conserved among SAM domains, are distinct from the specific Ste50p SAM amino acid mutations that differentially influence mating, filamentous growth, and osmotolerance in Saccharomyces cerevisiae (Jansen et al. 2001). However, the roles of the positionally equivalent tryptophan residues were not investigated in these other studies. The role of one of these positionally equivalent SAM mutations has been investigated in Ste50p; mutagenesis and gel filtration experiments indicate that V37 (equivalent to V23 in Ubc2) is not important for interaction with $S$. cerevisiae Ste11p (Grimshaw et al. 2004).

In this study, targeted yeast two-hybrid assays revealed that Ubc2 and Ubc4 heterodimerize via the SAM domains at their respective $\mathrm{N}$-termini, a result supported by in vitro co-immuno- 
precipitation analysis. This finding, and those of prior work (Mayorga and Gold 1998, 2001), indicate homology in the function of the Ste50p and Ubc2 proteins in pathways controlling mating and morphogenesis in $S$. cerevisiae and $U$. maydis, respectively. Similar to the situation described for Ste50p (Jansen et al. 2001), directed two-hybrid assays in this study indicated that $\mathrm{Ubc} 2$ does not homodimerize. Our two-hybrid analyses indicated that Ubc4 also does not homodimerize, which is in contrast to the reported ability of Stellp MAPKK kinase to homodimerize in the yeast system (Grimshaw et al. 2004; Jansen et al. 2001). As determined in this study, Ubc2 and Ubc4 only heterodimerize, although the possibility of undetectable transient homodimerization cannot be ruled out.

The presence of an RA domain (Ponting and Benjamin 1996) in Ubc2 suggests a potential interaction with Ras or another G-protein. In U. maydis, the Ras2 protein is known to function upstream of the MAP kinase pathway members Fuz7/Ubc5 and Ubc3/Kpp2 (Lee and Kronstad 2002). Moreover, Mst50 of $M$. grisea interacts with both Ras1 and Ras2 as determined in two-hybrid assays (Park et al. 2006). Thus, Ras 1 and Ras 2 of $U$. maydis represented candidates for interaction with Ubc2. In directed two-hybrid assays in this study, neither $U$. maydis Ras1 (Muller et al. 2003a) nor Ras2 (Lee and Kronstad 2002) exhibited a detectable interaction with Ubc2. Although Ubc2 may interact only transiently and weakly with Ras2, G-protein-binding at this site remains unknown. Although not tested in this study, other G-protein candidates for interaction with the Ubc2 RA domain include the Rho-type GTPases, including Cdc42, Rho, and Rac (Bishop and Hall 2000).

No potential interacting partners of the RA domain were uncovered in the directed two-hybrid assay in this study; the Ubc2 RA domain was determined to be essential for filamentous growth because combined mutations of three amino acids (D443A, D444A, and Y448A) in the RA domain prevented complementation to filamentous growth. The RA domain represents the region of amino acid sequence most highly conserved among the related adaptor proteins illustrated in Figure 1 and may indicate homology in function. In $S$. cerevisiae, the RA domain of Ste50p interacts specifically with Cdc42 Rho GTPase (Ramezani-Rad 2003; Truckses et al. 2006), a protein that is essential for appropriate pheromone-induced morphogenesis (Oehlen and Cross 1998). In the fission yeast Schizosaccharomyces pombe, the Byr2 MAPKK kinase, which is a homolog of Ste11p of Saccharomyces cerevisiae, contains a regulatory RA domain (Tu et al. 1997). It is through this RA domain that Byr2 interacts with Ras1 (Scheffzek et al. 2001; $\mathrm{Tu}$ et al. 1997) and functions downstream of Ras1 (Tu et al. 1997). A candidate RA domain has been identified in Ubc4 (Muller et al. 2003b). However, our directed two-hybrid assays in this study indicated that neither $U$. maydis Ras1 nor Ras2 proteins interact with the Ubc4 MAPKK kinase. Interacting partners of Ras2, providing a direct link to the pheromoneresponsive MAP kinase cascade in $U$. maydis, remain to be discovered.

Ubc2, unlike the other basidiomycete orthologous sequences, has a clear P-loop consensus near its C-terminus (Mayorga and Gold, 2001). However, deletion of this P-loop region generated no mutant phenotype in our assays. Additionally, because the motif is not conserved among the basidiomycetes, this region appears to play no conserved function.

Unlike Ste50p and its ascomycetous homologs, the Ubc2 adaptor protein possesses two C-terminal $\mathrm{SH} 3$ domains ( $\mathrm{SH} 3 \mathrm{a}$ and $\mathrm{SH} 3 \mathrm{~b}$ ) that are conserved in other basidiomycete orthologs (Fig. 1A), suggesting a novel role for this protein in signaling common to this group of fungi. SH3 domains are 50 to 70 amino acids in length, are commonly present in signal trans- duction and cytoskeletal regulatory proteins, and have a binding specificity for proline-rich sequences containing the core sequence PxxP, where $x$ is any amino acid (Kay et al. 2000). Although SH3 binding to proline-rich sequences is a general rule, it does not always apply (Agrawal and Kishan 2002). Aside from homology shared with other basidiomycete orthologs, sequence alignment of the $\mathrm{SH} 3 \mathrm{a}$ domain of $\mathrm{Ubc} 2$ reveals that the next closest ortholog is from a protein of unknown function (SPAC74) in Schizosaccharomyces pombe (Mayorga and Gold 2001). The Ubc2 SH3b domain most closely matches class $1 @$ SH3 domains, of which the Abl protein tyrosine kinase is a member and binds the consensus $@$ XxPxxP, where @ represents an aromatic or sometimes aliphatic residue (Cesareni et al. 2002).

Several SH3 domain-containing adaptor proteins have been identified and functionally characterized in fungi. The roles of these proteins are commonly associated with recruitment and regulation of kinase pathways controlling morphogenesis and mating. For example, Bem1p of Saccharomyces cerevisiae, a protein required for normal bud emergence and mating projection formation, contains two SH3 domains and interacts with numerous proteins, including $\mathrm{Cdc} 42 \mathrm{p}$ and its cognate guanine nucleotide exchange factor Cdc24p (Oehlen and Cross 1998). Bem1p also interacts with Boi2p, implicated in bud emergence and cell polarization, through its SH3-2 domain and a prolinerich site present in Boi2p (Matsui et al. 1996). The interaction of Bem1p and the Ste20p kinase also is mediated by the SH3-2 domain of Bem1p and a proline-rich motif present in the $\mathrm{N}$ terminal regulatory domain of Ste20p (Winters and Pryciak 2005). However, overall, given the lack of sequence similarity among the various $\mathrm{SH} 3$ domains and their binding properties (Cesareni et al. 2002), coupled with the complexity of the various SH3 domains in signaling, it is difficult to speculate on $\mathrm{SH} 3$ binding proteins for Ubc2. It is known that single amino acid changes can result in major differences in SH3 binding specificity or strength (Weng et al. 1995). In this study, mutagenesis of $u b c 2$ in only two codons specifying conserved amino acids in either $\mathrm{SH} 3 \mathrm{a}$ or $\mathrm{SH} 3 \mathrm{~b}$ generated strains that were completely nonpathogenic. These mutations likely diminished the binding of the Ubc2 $\mathrm{SH} 3$ domains to target proteins required for signaling.

As demonstrated in this study, both of the C-terminal SH3 domains of Ubc2 are essential for pathogenic development in U. maydis. This key and novel finding suggests a dual role for this protein in signaling (Fig. 6). Although the conserved Nterminus of Ubc2 is clearly essential for mating and filamentous growth as found for related adaptors in the Ascomycota, the basidiomycete-specific C-terminal SH3 domains play no role in these processes; however, they are necessary for pathogenicity. Although a functional mating response and filamentous growth are prerequisites for pathogenicity in wild-type $U$. maydis strains, meeting these prerequisites does not guarantee pathogenesis. For example, the $U$. maydis endoplasmic reticulum glucosidase II (gasl) mutant strain is normal in mating and growth but is arrested in growth in the plant epidermal layer (Schirawski et al. 2005), suggesting that other signaling components aside from the MAP kinase pathway are necessary for pathogenesis. Thus, it appears that the Ubc2-like adaptors may function as integrators of the conserved elements of the fungal pheromone responsive MAP kinase pathways and additional as yet uncharacterized signaling mechanisms specific to the Basidiomycota. Because the structure of the Ubc2 protein is conserved in ecologically distinct Basidiomycota, including both plant and animal pathogens as well as nonpathogenic species, further analyses of Ubc2-like proteins among the Basidiomycota will provide better understanding of its conserved role in signal transduction. 


\section{MATERIALS AND METHODS}

Fungal and bacterial strains and growth conditions.

Strains of $U$. maydis were grown at $30^{\circ} \mathrm{C}$ in liquid potato dextrose broth (PDB; Difco, Detroit) or on solid potato dextrose agar (PDA, Difco) supplemented to $2 \%$ agar (2PDA). The $U$. maydis strains used in this study are described in Table 1. U. maydis transformation was performed using a previously described method (Tsukuda et al. 1988). Transformed strains were grown on double complete agar medium with sorbitol (DCMS) and hygromycin B at $300 \mu \mathrm{g} / \mathrm{ml}$ (CalbiochemNovabiochem Corp., San Diego, CA, U.S.A.). At 5 to 10 days of growth on DCMS, individual transformants were transferred to PDA agar plates containing hygromycin B at $150 \mu \mathrm{g} / \mathrm{ml}$ (Calbiochem-Novabiochem). Carboxin (Vitavax 34; Gustafson, Dallas) was used in selection medium at a final concentration of $3 \mu \mathrm{g} / \mathrm{ml}$. Mating assays were done on charcoal mating plates made of double complete agar medium (DCM) containing 1\% activated charcoal (Holliday 1974).

Escherichia coli strains XL-1 Blue (Stratagene, La Jolla, CA, U.S.A.), DH5 $\alpha$ and DH10B (Bethesda Research Laboratories, Gaithersburg, MD, U.S.A.), used in DNA manipulations, were grown in liquid Luria-Bertani (LB) medium or on LB agar plates with the appropriate antibiotics.

\section{Phylogenetic analysis of Ubc2-type adaptors.}

The phylogenetic tree was generated from a pairwise distance matrix using Phylogenetic Analysis Using Parsimony (PAUP), version 4.0b10 for Macintosh (PPC/Altivec). The starting trees were obtained via neighbor joining and support for the tree branching was obtained using a bootstrap approach with 100 replicate trees. The input for the tree was obtained from the multiple sequence alignment (CLUSTALW) of the SAM (amino acids 16 to 93 ) and RA (amino acids 416 to 490) region of Ubc2 (accession AAK49432) with the trimmed SAM and RA domain regions of $C$. neoformans (accession AAW42725), Yarrowia lipolytica (accession XP_504137), A. oryzae (accession BAE61211), Coccidioides immitis (accession EAS28206), A. nidulans (accession XP_680521), Chaetomium globosum (accession EAQ91188), A. fumigatus (accession AAM08676), Neurospora crassa (accession XP_956774), M. grisea (accession XP_359578), Gibberella zeae (accession XP_384277), Candida albicans (accession XP_721713), Kluyveromyces lactis (accession XP_452257), Debaryomyces hansenii (accession XP_459247), Schizosaccharomyces pombe (accession CAB38684), C. glabrata (accession XP_445008), and Saccharomyces cerevisiae (accession NP_009898). Each of the protein accessions listed above were derived from the National Center for Biotechnology Information identified in BLASTP (Altschul et al. 1997) searches. Additional putative Ubc2 adaptor protein orthologs are encoded at locus RO3G_03290.1 in the R. oryzae Sequencing Project database and at locus CC1G_00975.1 in the Coprinus cinereus Sequencing Project database, Broad Institute of Harvard and MIT. The predicted orthologous protein for Phanerochaete chrysosporium (protein ID 2321 encoded at location: Phchr1/scaffold_3:1013644-1016065) was obtained from sequence data produced by the United States Department of Energy, Joint Genome Institute.

\section{Construction of $u b c 2$ mutant alleles.}

All site-directed mutagenesis reactions were performed with the Quickchange Site-Directed Mutagenesis Kit (Stratagene) according to the manufacturer's instructions using $10 \mathrm{ng}$ of pSAC7 (Mayorga and Gold 1998) as template. Sense and antisense primer combinations were designed to introduce amino acid changes or deletions in the regions encoding the various putative protein interaction domains of $u b c 2$ and are listed in Table 4. All mutant alleles were confirmed by sequencing.

The deletion construct of the region encoding the $u b c 2$ C-terminus was generated by overlap PCR (Davidson et al. 2002). In all PCR steps, the Expand High Fidelity (Roche, Branchburg, NJ, U.S.A.) Taq polymerase was used according to the manufacturer's instructions. Primer pairs SK056 and SK058 (Table 5) were designed to amplify approximately $1,000 \mathrm{bp}$ of $u b c 2,5^{\prime}$ of the end of the region encoding the RA domain, and to introduce two stop codons immediately $3^{\prime}$ of the RA domain. The SK058 primer also was designed to overlap the $5^{\prime}$ end of a $2.3-\mathrm{kb}$ carboxin resistance $\left(c b x^{R}\right)$ gene fragment. The $c b x^{R}$ fragment was amplified using overlap primers SK057 and SK060. Primers SK059, containing a $3^{\prime} c b x^{R}$ overlap, and SK061 were designed to amplify 1,000 bp flanking the $3^{\prime}$ end of the $u b c 2$ open reading frame (ORF). In all, $10 \mathrm{ng}$ of each of the two 1-kb flanking PCR products, along with $25 \mathrm{ng}$ of the $2.3-\mathrm{kb} c b x^{R}$ product, were combined and used as template for the first round of PCR. In the second round of PCR, primers SK056 and SK061 were added to amplify the entire 4.3-kb overlap PCR product, which was cloned in vector pCR2.1 (Invitrogen). The resultant construct was released from pCR2.1 (Invitrogen) with $E c o \mathrm{R} 1$ and used for transformation.

For filamentation complementation experiments, wild-type and mutant versions of $u b c 2$ in plasmid pSAC7 (and its mutated derivatives) were transformed into the budding uacl $u b c 2$ double mutant strain, 5/17 (Mayorga and Gold 1998). A
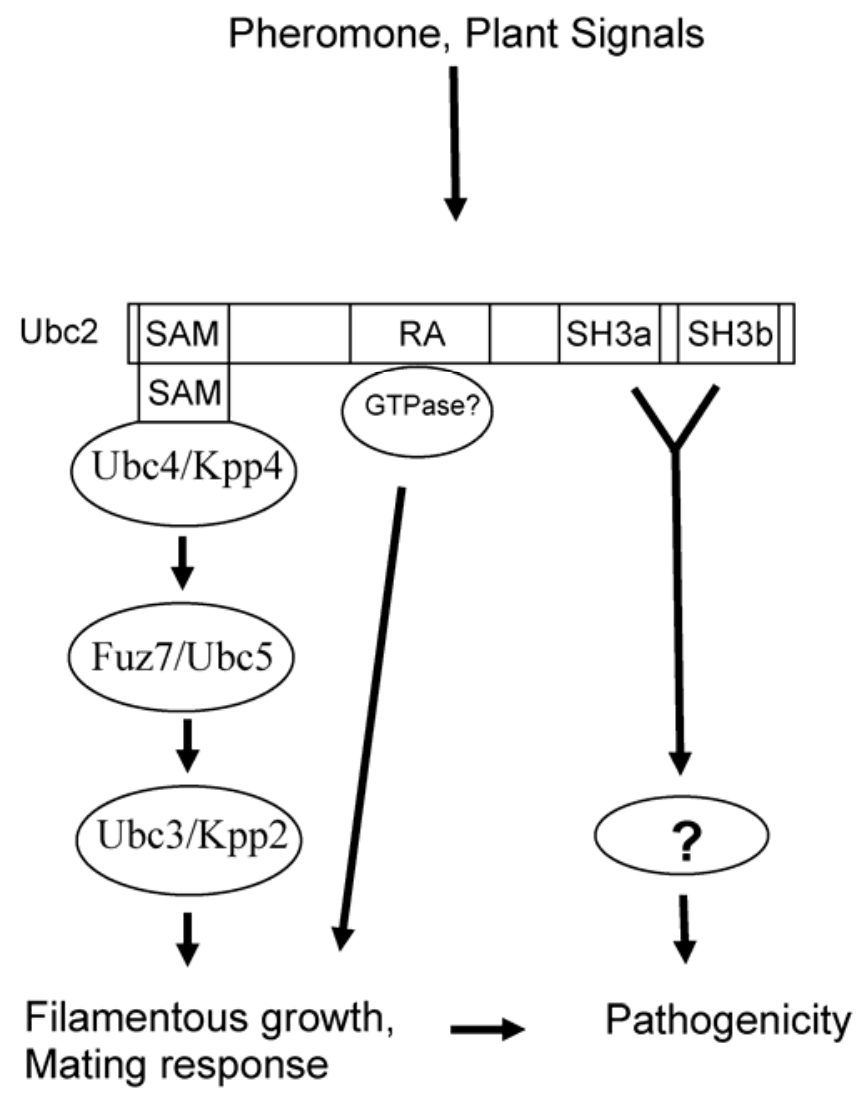

Fig. 6. Model of Ubc2 function in Ustilago maydis. The Ubc2 adaptor protein facilitates the coupling of environmental/genetic signals to signal transduction via conserved protein-protein interaction domains, likely forming a multiprotein complex. The Ubc2 sterile- $\alpha$-motif (SAM) domain interacts directly with the pheromone responsive mitogen-activated protein kinase cascade via binding the SAM domain of Ubc4. The ras association (RA) domain influences filamentous growth through undetermined mechanisms. A key finding of this report is that, via its two src homology 3 (SH3) domains and its unknown binding partners, the C-terminus of Ubc2 signals for pathogenicity independent of the morphogenetic pheromone response. 
separate construct was produced to determine whether the $u b c 2$ C-terminal deletion construct could complement the $u b c 2$ mutant for filamentation. For this construct, SacI linker primers SK084 and SK091 were used to amplify the promoter region of $u b c 2,5^{\prime}$ of the start site to the point immediately $3^{\prime}$ of the region encoding the RA domain. Primer SK091 included two stop codons that previously were introduced in plasmid pSAC7 by mutagenesis. The PCR product was subcloned into pCR2.1 (Invitrogen), cut with SacI, ligated into the SacI site of pHyg101 (Mayorga and Gold 1998), and transformed into strain $5 / 17$. To be certain that no unexpected changes were introduced into the two mutant pSAC7 derivative plasmids WVW and DDY (Table 2) that were incapable of complementing for filamentation, we sequenced across the promoter and ORF in the construct of each. In each case, the promoter region and ORF were unchanged except for the desired mutagenized regions.

For complementation of defective pathogenicity function, the ARS was removed from pSAC7 and mutant derivatives and the resultant linearized plasmids, containing either wild-type or mutant $u b c 2$ alleles, were transformed into the compatible C-terminal deletion strains $15 / 9$ and $15 / 10$. The resulting transformants were screened by PCR for the presence of the appropriate construct and used for plant inoculations. To be certain that no unexpected changes were introduced in the two pSAC7 derivative plasmids $(\Delta \mathrm{SH} 3 \mathrm{a}$ and $\Delta \mathrm{SH} 3 \mathrm{~b})$ that bore deletion mutations in the SH3 domains and were incapable of complementing for pathogenicity function, we sequenced across their respective promoters and ORFs in the construct. In each case, the promoter region and ORF were unchanged except for the intended deleted segments in the SH3 domains.

\section{DNA blot analysis.}

To confirm the genomic deletion of the region encoding the C-terminus of $u b c 2$ in strains $15 / 9$ and 15/10, a DNA blot was performed. U. maydis wild-type and transformant genomic DNA was isolated and digested with HindIII. Approximately $10 \mu \mathrm{g}$ of DNA was loaded in each lane of a $1 \%$ agarose gel and transferred to a Zeta Probe membrane (BioRad, Hercules, CA, U.S.A.) by alkaline transfer. Prehybrization and hybridization conditions were performed following the manufacturer's recommendations. A DNA probe of wild-type $u b c 2$ containing flanking regions was generated with primers SK089 and SK090 (Table 5) and digoxigenin-labeled using the DIG-High Prime kit (Roche) following the manufacturer's instructions (Roche).

\section{Mating analyses.}

Following overnight growth in liquid culture, U. maydis strains were mixed in equal volumes and $8 \mu \mathrm{l}$ of each cell mix- ture was plated onto charcoal mating plates (Holliday 1974). The plates were incubated at room temperature in the dark and assessed for the presence of white filamentous growth indicative of mating after $48 \mathrm{~h}$.

\section{Pathogenicity assays.}

Pathogenicity assays were performed as described previously (Gold et al. 1997). Seven-day-old Golden Bantam maize seedlings were grown and inoculated in a growth chamber (Conviron, model E15) with 12-h night and day cycles at $30^{\circ} \mathrm{C}$ constant and assessed for symptom development at 7, 10, 14, and 21 days following inoculation. The initial inoculum concentration was $1 \times 10^{6}$ cells $/ \mathrm{ml}$ for each strain. All experiments were repeated at least three times.

\section{Yeast two-hybrid studies.}

Each of the ORFs encoding Ubc2, Ubc3, Ubc4, Ras1, and Ras2 were amplified using restriction enzyme linker primers and Expand high-fidelity Taq polymerase (Roche) according to the manufacturer's instructions and cloned into pCR2.1 (Invitrogen). Inserts subsequently were cut from pCR2.1 with the appropriate restriction enzyme and ligated in frame with the GAL4 activation domain of prey plasmid pGADT7 (BD Biosciences, Franklin Lakes, NJ, U.S.A.). Primer pairs for amplification of $u b c 4$ were SK013 and SK014 (Table 5). Primers for

Table 5. Additional primers used in this study

\begin{tabular}{ll}
\hline Primer & \multicolumn{1}{c}{ Sequence $\left(\mathbf{5}^{\prime} \rightarrow \mathbf{3}^{\prime}\right)$} \\
\hline SK013 & GAATTCATGAGTGCTGCAACACCTACC \\
SK014 & CTAAGAATCCGCCTCTTCG \\
SK025 & CGCGAATTCATGAGTGGCAAAATGATGATCTAC \\
RasXhoI & CTCGAGTCAAAGGATATTGCATTTGA \\
SK026 & GAGGAATTCATGTCCAAAGCACAATTCTTGCG \\
SK027 & GTTAGAGAACGATACATTTCTGGC \\
SK033 & GCGAATTCATGTCCTCTCAAAGCCCAAAG \\
SK034 & GTCGCGGATGTGACGAAGC \\
SK035 & CTTGAGACGGTAGATGGCAGC \\
SK036 & TCAGACGGTAGCCATCGGCGC \\
SK039 & CCGAATTCGCATCCTCCGACAGCAACCCG \\
SK050 & CGAATTCGTTGAGCAGCTTGATGGC \\
SK056 & ATTCCATTGACACGCCCATC \\
SK057 & ACATCCGCGACGTATAATAGGTCAGGTTGTGGTCGACGCC \\
SK058 & GGCGTCGACCACAACCTGACCTATTATACGTCGCGGATGT \\
SK059 & CGAAAGCGAGACGAGTTGAGCCTCGACGTGCCTCAGGTGAC \\
SK060 & GTCACCTGAGGCACGTCGAGGCTCAACTCGTCTCGCTTTCG \\
SK061 & AACCGCAACCATCGCAAC \\
SK084 & GAGCTCCTTCCAACGGCACGATCGAC \\
SK089 & CAATCGAGGCGCAAGAACATG \\
SK090 & CTTCCAATCACTCGCTGACGG \\
SK091 & GAGCTCCTATTATACGTCGCGGATGTGACG \\
\hline
\end{tabular}

Table 4. Primers used in site-directed and deletion mutagenesis of $u b c 2$ in this study

\begin{tabular}{|c|c|c|c|}
\hline Primer & Sequence $\left(5^{\prime} \rightarrow 3^{\prime}\right)$ & $u b c 2$ Codon changes & Domain $^{a}$ \\
\hline WVW & GTCAACAAGGCGTCCGAGCAGCAGGCCGTAGACGCGCTCTCCTCCG & W17A, V23A, W25A & SAM \\
\hline KRD & CAACCCGTACGCGTCGTTTGCCGTCACTCTCGACGCCCCATGCTAT & K418A,R421A,D426A & RA \\
\hline DDY & CAAGATCAACGCCGCCTGGCGCAAGGCCGCGCTCTTTATC & D443A,D444A,Y448A & RA \\
\hline EEP & GGCAAGACGGCGCGGTGTCTCAGCTACGACGCAAAAGCTCTGCTTC & E458A,E465A, P467A & RA \\
\hline YY & GTGGCCATCGCCCCAGCCATGCCCGAG & Y572A, Y574A & SH3a \\
\hline GD & GTGAATGTGGCCGATACGTTTGTC & G587A, D588A & SH3a \\
\hline LK2 & CGAGGGCGATGCGGATCTGCGCGCGGACGAGCGTC & L725A, K729A & SH3b \\
\hline GP2 & GCACGCCAGGGCCTGGGTCGCCAGCTGGTAC & G755A, P758A, & $\mathrm{SH} 3 \mathrm{~b}$ \\
\hline SY2 & CTGGGTCCCCGCCTGGGCCATTGGCAAGC & S759A, Y761A & SH $3 b$ \\
\hline P-all & GCCAGCGGATCGTTGGCCGCGGCGGCGTCGACCACTG & G774A,K775A,S776A & P-loop \\
\hline SK019 & CCGCGACGTATAATAGCCCATTGCG & K492Stop, S493Stop & C-terminus \\
\hline SK127 & GCGAGAACATACGCGGTGGCCATCGAGTTTGACGTGAATGTGGGCATAC & Delete $572-580$ & $\mathrm{SH} 3 \mathrm{a}$ \\
\hline SK129 & GTACAGGAGCGAGATGCGCACGCCAAGCTTTCCTCGGCCGCCAGCGG & Delete $754-763$ & SH3b \\
\hline SK113 & TTGGCAAGCTTTCCTCGGCCGCGTCGACCACTGCTTTGTC & Delete $769-776$ & P-loop \\
\hline
\end{tabular}

${ }^{\text {a }} \mathrm{SAM}=$ sterile- $\alpha$-motif, $\mathrm{RA}=$ ras association, and $\mathrm{SH} 3=\operatorname{src}$ homology 3 domains . 
ras1 were SK026 and SK027. Primers for ras2 were SK025 and RasXho1. The $u b c 5$ ORF was manipulated similarly, except BamHI linker primers were used in the amplification and ligated in frame into the BamHI site of pGADT7. Primers for amplifying the SAM domain region of the $u b c 4$ ORF were SK013 and SK050. The RASH3 truncated version of $u b c 2$ was amplified with SK036 and SK039. The SAM region was amplified with primers SK033 and SK035 and the SAMRA clone with SK033 and SK034. The $u b c 2$ and $u b c 4$ ORFs also were amplified with EcoR1 linker primers, cloned into pCR2.1, cut with $E c o$ R1, and ligated into the EcoR1 site of the bait plasmid pGBKT7. All constructs were sequenced across the polylinker-start codon junction to verify correct reading frame.

The Matchmaker GAL4 Two-Hybrid System 3 (BD Biosciences) was used to perform all directed two-hybrid assays. Bait and prey plasmid constructs were introduced into $S$. cerevisiae strain AH109 (BD Biosciences) by cotransformation. Growth was assessed after 5 days on both -Trp/-Leu/-His SD medium supplemented with $10 \mathrm{mM}$ 3-amino-1,2,4-triazole (3AT; Sigma. St. Louis) and -Trp/-Leu/-His/-Ade synthetic dropout medium. The latter medium was overlayed with $\mathrm{X}-\alpha-\mathrm{Gal}$ (BD Biosciences). The amount of 3-AT used was determined by assessing growth of positive controls in the presence of 10 and $30 \mathrm{mM}$ concentrations of $3-\mathrm{AT} ; 30 \mathrm{mM}$ was deemed slightly inhibitory to the growth of positive controls whereas both concentrations were fully inhibitory to negative control strains. Therefore, $10 \mathrm{mM} 3$-AT was employed. Immunoblotting was performed to verify that prey and bait fusion proteins were expressed in yeast. The protein extracts were processed from the experimental yeast strains assayed for two-hybrid interactions.

For co-immunoprecipitation, the pGBKT7 bait plasmids and the pGADT7 prey plasmids containing the ORF inserts in frame with the c-Myc and HA tags, respectively, were isolated from $E$. coli by alkaline lysis and precipitated twice with $95 \%$ ethanol to remove residual RNase A. Each of the plasmids was further purified on a Qiagen column before in vitro transcription and translation using the TNT T7-Coupled Transcription/ Translation system (Promega, Madison, WI, U.S.A.). The matchmaker CO-IP kit (BD Biosciences) was used in accordance with the manufacturer's instructions.

\section{Immunoblots.}

Yeast protein extracts were prepared according to the urea/ sodium dodecyl sulfate (SDS) method (Printen and Sprague 1994). The extracts were electrophoresed on an 8 to $16 \%$ SDSpolyacrylamide gel (Gradiopore, North Ryde, Australia) and transferred to an enhanced chemiluminescence (ECL) nitrocellulose membrane (Amersham-Pharmacia, Piscataway, NJ, U.S.A.), and detection was performed following the manufacturer's instructions. Membranes were incubated with HRPconjugated secondary antibodies (BD Biosciences) at a 1:5,000 dilution in blocking buffer for $1 \mathrm{~h}$. Membranes were washed three times for $10 \mathrm{~min}$ in phosphate-buffered saline and $0.2 \%$ tween-20, and detection was performed with an ECL system (Amersham-Pharmacia) following the manufacturer's instructions.

\section{DNA sequencing and analysis.}

Sequencing reactions were carried out in our laboratory using the Big Dye (v3.1) Terminator Cycle Sequencing kit (Applied Biosystems, Foster City, CA, U.S.A.) following the manufacturer's instructions and run on an ABI Prism 310 Capillary Automated Genetic Analyser (Applied Biosystems) at the Genome Analysis Facility on site. DNA sequences were analyzed with MacDNAsis software (v.3.5; Hitachi Software Engineering Co. Ltd., Tokyo).

\section{ACKNOWLEDGMENTS}

This work was supported by the United States Department of Agriculture National Research Initiative Competitive Grants nos. 20013531910139 and 20033531913361 to S. E. Gold and no. 20033531913471 to S. J. Klosterman. We thank F. Martin for assistance in using PAUP to generate the distance tree and L. Szabo for providing P. graminis f. sp. tritici sequences prior to publication.

\section{LITERATURE CITED}

Agrawal, V., and Kishan, K.V. 2002. Promiscuous binding nature of SH3 domains to their target proteins. Protein Pept. Lett. 9:185-193.

Altschul, S. F., Madden, T. L., Schaffer, A. A., Zhang, J., Zhang, Z., Miller, W., and Lipman, D. J. 1997. Gapped BLAST and PSI-BLAST: A new generation of protein database search programs. Nucleic Acids Res. 25:3389-3402.

Andrews, D. L., Egan, J. D., Mayorga, M. E., and Gold, S. E. 2000. The Ustilago maydis $u b c 4$ and $u b c 5$ genes encode members of a MAP kinase cascade required for filamentous growth. Mol. Plant-Microbe Interact. 13:781-786.

Banuett, F., and Herskowitz, I. 1994. Identification of fuz7, a Ustilago maydis MEK/MAPKK homolog required for a-locus-dependent and independent steps in the fungal life cycle. Genes Dev. 8:1367-1378.

Barr, M. M., Tu, H., Van Aelst, L., and Wigler, M. 1996. Identification of Ste4 as a potential regulator of Byr2 in the sexual response pathway of Schizosaccharomyces pombe. Mol. Cell Biol. 16:5597-5603.

Bishop, A. L., and Hall, A. 2000. Rho GTPases and their effector proteins. Biochem. J. 348:241-255.

Cesareni, G., Panni, S., Nardelli, G., and Castagnoli, L. 2002. Can we infer peptide recognition specificity mediated by SH3 domains? FEBS (Fed. Eur. Biochem. Soc.) Lett. 513:38-44.

Davidson, R. C., Blankenship, J. R., Kraus, P. R., de Jesus Berrios, M., Hull, C. M., D’Souza, C., Wang, P., and Heitman, J. 2002. A PCR-based strategy to generate integrative targeting alleles with large regions of homology. Microbiology 148:2607-2615.

Gold, S., Duncan, G., Barrett, K., and Kronstad, J. 1994. cAMP regulates morphogenesis in the fungal pathogen Ustilago maydis. Genes Dev. 8:2805-2816.

Gold, S. E., Brogdon, S. M., Mayorga, M. E., and Kronstad, J. W. 1997. The Ustilago maydis regulatory subunit of a cAMP-dependent protein kinase is required for gall formation in maize. Plant Cell 9:1585-1594.

Grimshaw, S. J., Mott, H. R., Stott, K. M., Nielsen, P. R., Evetts, K. A., Hopkins, L. J., Nietlispach, D., and Owen, D. 2004. Structure of the sterile alpha motif (SAM) domain of the Saccharomyces cerevisiae mitogen-activated protein kinase pathway-modulating protein STE50 and analysis of its interaction with the STE11 SAM. J. Biol. Chem. 279:2192-2201.

Holliday, R. 1974. Ustilago maydis. In: Handbook of Genetics. R. C. King, ed. Plenum, New York.

Jansen, G., Buhring, F., Hollenberg, C. P., and Rad, M. R. 2001. Mutations in the SAM domain of STE50 differentially influence the MAPK-mediated pathways for mating, filamentous growth and osmotolerance in Saccharomyces cerevisiae. Mol. Genet. Genomics 265:102-117.

Kaffarnik, F., Muller, P., Leibundgut, M., Kahmann, R., and Feldbrugge, M. 2003. PKA and MAPK phosphorylation of Prf1 allows promoter discrimination in Ustilago maydis. EMBO (Eur. Mol. Biol. Organ.) J. 22:5817-5826.

Kay, B. K., Williamson, M. P., and Sudol, M. 2000. The importance of being proline: The interaction of proline-rich motifs in signaling proteins with their cognate domains. FASEB (Fed. Am. Soc. Exp. Biol.) J. 14:231-241.

Lee, N., and Kronstad, J. W. 2002. ras 2 controls morphogenesis, pheromone response, and pathogenicity in the fungal pathogen Ustilago maydis. Eukaryot. Cell 1:954-966.

Mayorga, M. E., and Gold, S. E. 1998. Characterization and molecular genetic complementation of mutants affecting dimorphism in the fungus Ustilago maydis. Fungal Genet. Biol. 24:364-376.

Mayorga, M. E., and Gold, S. E. 1999. A MAP kinase encoded by the $u b c 3$ gene of Ustilago maydis is required for filamentous growth and full virulence. Mol. Microbiol. 34:485-497.

Mayorga, M. E., and Gold, S. E. 2001. The ubc2 gene of Ustilago maydis encodes a putative novel adaptor protein required for filamentous growth, pheromone response and virulence. Mol. Microbiol. 41:13651379

Muller, P., Aichinger, C., Feldbrugge, M., and Kahmann, R. 1999. The MAP kinase kpp2 regulates mating and pathogenic development in Ustilago maydis. Mol. Microbiol. 34:1007-1017.

Muller, P., Katzenberger, J. D., Loubradou, G., and Kahmann, R. 2003a. 
Guanyl nucleotide exchange factor $\mathrm{Sq} 12$ and Ras2 regulate filamentous growth in Ustilago maydis. Eukaryot. Cell 2:609-617.

Muller, P., Weinzierl, G., Brachmann, A., Feldbrugge, M., and Kahmann, R. 2003b. Mating and pathogenic development of the smut fungus Ustilago maydis are regulated by one mitogen-activated protein kinase cascade. Eukaryot. Cell 2:1187-1199.

Oehlen, L. J., and Cross, F. R. 1998. The role of Cdc42 in signal transduction and mating of the budding yeast Saccharomyces cerevisiae. J. Biol Chem. 273:8556-8559.

Park, G., Xue, C., Zhao, X., Kim, Y., Orbach, M., and Xu, J. R. 2006. Multiple upstream signals converge on the adaptor protein Mst50 in Magnaporthe grisea. Plant Cell 18:2822-2835.

Ponting, C. P. 1995. SAM: A novel motif in yeast sterile and Drosophila polyhomeotic proteins. Protein Sci. 4:1928-1930.

Ponting, C. P., and Benjamin, D. R. 1996. A novel family of Ras-binding domains. Trends Biochem. Sci. 21:422-425.

Printen, J. A., and Sprague, G. F., Jr. 1994. Protein-protein interactions in the yeast pheromone response pathway: Ste $5 p$ interacts with all members of the MAP kinase cascade. Genetics 138:609-619.

Ramachander, R., Kim, C. A., Phillips, M. L., Mackereth, C. D., Thanos, C. D., McIntosh, L. P., and Bowie, J. U. 2002. Oligomerization-dependent association of the SAM domains from Schizosaccharomyces pombe Byr2 and Ste4. J. Biol. Chem. 277:39585-39593.

Ramezani-Rad, M. 2003. The role of adaptor protein Ste50-dependent regulation of the MAPKKK Ste11 in multiple signalling pathways of yeast. Curr. Genet. 43:161-170.

Scheffzek, K., Grunewald, P., Wohlgemuth, S., Kabsch, W., Tu, H., Wigler, M., Wittinghofer, A., and Herrmann, C. 2001. The Ras-Byr2RBD com- plex: Structural basis for Ras effector recognition in yeast. Structure 9:1043-1050.

Schirawski, J., Bohnert, H. U., Steinberg, G., Snetselaar, K., Adamikowa, L., and Kahmann, R. 2005. Endoplasmic reticulum glucosidase II is required for pathogenicity of Ustilago maydis. Plant Cell 17:3532-3543.

Truckses, D. M., Bloomekatz, J. E., and Thorner, J. 2006. The RA domain of Ste 50 adaptor protein is required for delivery of Ste 11 to the plasma membrane in the filamentous growth signaling pathway of the yeast Saccharomyces cerevisiae. Mol. Cell Biol. 26:912-928.

Tsukuda, T., Carleton, S., Fotheringham, S., and Holloman, W. K. 1988. Isolation and characterization of an autonomously replicating sequence from Ustilago maydis. Mol. Cell Biol. 8:3703-3709.

Tu, H., Barr, M., Dong, D. L., and Wigler, M. 1997. Multiple regulatory domains on the Byr2 protein kinase. Mol. Cell Biol. 17:5876-5887.

Weng, Z., Rickles, R. J., Feng, S., Richard, S., Shaw, A. S., Schreiber, S. L., and Brugge, J. S. 1995. Structure-function analysis of SH3 domains: SH3 binding specificity altered by single amino acid substitutions. Mol. Cell Biol. 15:5627-5634.

Zhao, X., Kim, Y., Park, G., and Xu, J. R. 2005. A mitogen-activated protein kinase cascade regulating infection-related morphogenesis in $\mathrm{Mag}$ naporthe grisea. Plant Cell 17:1317-1329.

\section{AUTHOR-RECOMMENDED INTERNET RESOURCES}

Broad Institute of Harvard and MIT website: www.broad.mit.edu United States Department of Energy, Joint Genome Institute website: www.jgi.doe.gov 